\title{
Construction du savoir langagier en français à la Légion étrangère : la double hybridation linguistique dans l'interlangue des légionnaires russes et polonais
}

Héléna MANIAKIS, Université Lyon 2

\section{Introduction}

Créée par une ordonnance de Louis-Philippe en 1831, la Légion étrangère est une particularité de l'armée française. Actuellement, elle regroupe en son sein plus de centquarante nationalités. Pour la plupart des militaires servant à titre étranger, la finalité de l'engagement est l'acquisition de la nationalité française et l'insertion dans la vie civile. Cette insertion dépend en grande partie de leur aisance en français. Lors de l'instruction du jeune légionnaire, il incombe à la Légion la tâche d'enseigner le français à toutes les recrues dans le délai le plus court possible : il en va de la cohésion du groupe et de l'efficacité de l'exécution des ordres reçus par les recrues. Le travail présenté ici se concentre sur les acquisitions en français des légionnaires russes et polonais. Pour aborder le français parlé à la Légion, il convient d'aborder en premier lieu le mode d'apprentissage de la langue dans ce contexte si particulier. Dans une seconde partie, nous tenterons une définition de la langue parlée à la Légion étrangère: comment définir au mieux cet hybride linguistique? Enfin, nous présenterons quelques corpus, afin de mettre en évidence une double hybridation : l'impact de la première langue en premier lieu, et, ensuite, l'existence de structures stéréotypées, preuves de la seconde hybridation : le légiolecte.

\section{Le contexte d'acquisition à la Légion étrangère}

\subsection{Un contexte plurilingue et une communication exolingue}

L'acquisition du français à la Légion étrangère se fait dans un contexte plurilingue, où le français a vocation à devenir langue véhiculaire et où, pour optimiser son apprentissage, il est interdit aux légionnaires de parler leur première langue. La communication avec les gradés et les recrues francophones est une communication exolingue, si l'on retient la définition d'interaction exolingue comme une asymétrie entre les répertoires et/ou les compétences des participants (Porquier). L'interaction exolingue peut donc aussi bien caractériser une communication entre deux locuteurs n'ayant pas le même niveau de compétence dans une langue, mais également entre deux locuteurs ayant un niveau d'expertise différent dans un domaine donné. Le légionnaire venant d'un métier civil ou n'ayant jamais servi sous les drapeaux dans son pays cumule donc les deux caractéristiques. 
La communication est, de surcroît, asymétrique entre gradés et jeunes recrues. Or, si l'acquisition $\mathrm{du}$ français est primordiale pour le commandement au niveau de la compréhension des ordres, elle est tout aussi importante pour le légionnaire : il en va de son avancement, de sa réussite de certains stages et, surtout, de sa naturalisation. Jusqu'ici, la Légion étrangère gérait elle-même les dossiers de naturalisation et faisait donc passer à ces fins des tests de français de son cru. Depuis fin 2012, une restructuration importante a lieu et la Légion s'alignera à terme aux exigences du civil, à savoir le niveau B1 oral pour l'acquisition de la nationalité française. Nous avons pu accéder à ces tests. C'est le test de niveau 3 qui est requis pour la naturalisation, et les compétences à valider sont énoncées sur la première page.

\subsection{La méthode d'acquisition : la méthode « Képi blanc »}

«Si ça rentre pas par les oreilles, ça rentrera par les bras. »

C'est lors de l'instruction du jeune légionnaire au régiment de Castelnaudary que se joue le plus important de l'apprentissage du français pour les recrues. Le régiment sert de base, mais l'instruction se déroule dans un lieu-dit «la ferme », mot qui résonne bien particulièrement pour les jeunes engagés volontaires, et qui ne leur laisse pas les meilleurs souvenirs. Il y a en réalité plusieurs fermes, comme nous l'avons constaté lors d'une journée d'observation. Au terme des quatre mois d'instruction, les légionnaires sont supposés avoir acquis cinq cents mots. Il s'agit d'une acquisition guidée dans laquelle, en théorie, les légionnaires doivent être placés en trinômes: deux alloglottes pour un francophone, ce dernier étant chargé de leur enseigner tout ce qui est nécessaire pour comprendre les ordres. Dans la pratique, il y a bien souvent trop peu de francophones et les récits des légionnaires que j'ai pu interviewer, font plutôt état d'un francophone pour quatre à six alloglottes. Les francophones s'improvisent donc enseignants de français. Si le légionnaire francophone est doué pour enseigner, il se voit parfois confier jusqu'à sept légionnaires. Sur la totalité de la durée de l'instruction, le commandement estime le volume horaire des cours de français en classe, dispensés cette fois par un gradé, à soixante-dix-huit heures. C'est à Castelnaudary que l'input linguistique est le plus intense et l'injonction de parler français la plus forte. Ce contexte crée l'identité du légionnaire par la transmission des traditions et par la langue véhiculaire que devient le français : on fait du français dans la salle de cours et à tout instant de la journée.

Cette méthode d'acquisition est cependant loin d'être celle dispensée dans les centres spécialisés dans l'acquisition du français aux étrangers, comme l'attestent les témoignages que j'ai recueillis. Parmi toutes mes interviews, s'en trouve une particulièrement précieuse, 
car son auteur fait preuve de recul et d'une analyse réflexive sur l'instruction à Castelnaudary. Il s'agit des propos de A., Français engagé volontaire après quelques années sur les bancs de l'université. Devant son implication et son application avec ses alloglottes durant l'instruction, ses supérieurs lui en confient sept :

«Sept. Deux Ukrainiens, un Russe, un Lettonien, un Espagnol et un Chinois et le dernier j'me souviens plus, j'crois il était roumain. Bon le Roumain ça va tout seul. Et en fait on a les cours de français obligatoires, bien sûr si ils répondent faux ou si ils savent pas répondre, si ils s'endorment, si ils montrent pas d'intérêt pour apprendre le cours, les punitions, elles sont pas par exemple euh tu dois écrire euh cent fois l'truc, c'est des punitions physiques. Bon, quand tu rentres encore plus crevé, comment tu peux t'attendre... du genre fais vingt pompes si t'as pas réussi à conjuguer le verbe être [...]. On peut pas dire que quelqu'un de fatigué, d'épuisé, qui vient de pomper il apprendra mieux le verbe être. Bah il est un peu humilié, parce qu'il a pompé devant tout le monde, mais au contraire, ça le rend aigri par rapport au cours, ça va pas l'inciter à s'impliquer davantage. »

Méthode d'enseignement confirmée par J., Monténégrin, à peine sorti de l'instruction (huit mois de service au moment de l'interview) :

"Lieutenant il s'énervait toujours parce que un Népalais il n'arrive pas dire normalement et le lieutenant il dit 'ça c'est le mur', 'ça c'est le mur', 'ça c'est le tableau'. 'ça c'est le tableau', 'ça c'est la bouche', 'ça, c'est la bousse', 'ça c'est la bouche', 'ça, c'est la bousse' après il commence à répéter, répéter, vingt, trente fois, après tout le monde qui commence à rigoler il mettait tout le monde en position, il restait debout avec Népalais comme ça trente quarante minutes chaque jour. (Pause). Et il a jamais arrivé. »

Les autres contacts principaux du légionnaire avec la langue française lors de l'input initial sont le carnet de chant et le code du légionnaire. Le code du légionnaire doit être appris par cœur le plus vite possible, de même que certains chants : l'apprentissage se fait ici à la va-vite, sans compréhension fine des textes, et le plus souvent avec une transcription phonétique des sons du français avec l'alphabet de la première langue. Depuis fin 2013, un changement notable a eu lieu : le code d'honneur du légionnaire est traduit dans la première langue des recrues.

Il ne s'agit pas ici de jeter la pierre aux gradés, dont la tâche est un défi. En effet, ils n'ont ni les outils ni la formation suffisante que requiert l'enseignement du français à des étrangers, et les lieux ne sont pas faits pour apprendre la langue. Mais il est légitime de s'interroger sur le bien-fondé des «bonnes vieilles méthodes » avec punitions physiques à la clé. Le cours de français auquel il m'a été donné d'assister dans une des «fermes» de Castelnaudary, était dispensé par un lieutenant un peu décontenancé par sa tâche, qui m'a confié : «Moi je pensais pas quand j'étais au collège et au lycée, moi, dyslexique et ancien 
bègue, me retrouver à faire des cours de français ». C'est dans ce contexte plurilingue, exolingue, et aux méthodes particulières qu'émerge le français de la Légion.

\section{Pidgin, lecte d'apprenant, interlangue ? Définir la langue parlée à la Légion}

"Alors c'est un mélange de slang français, de mots de d'autres langues, avec une grammaire simplifiée, avec un accent étrangère, et là ça donne euh... quelque chose énorme »(A., Russe, 15 ans de service).

La communication exolingue évoquée plus haut n'est pas sans conséquence sur le type de langage appris à la Légion. En effet, si le français est la langue véhiculaire imposée, et si les répertoires linguistiques sont différents, alors il s'agit d'une communication unilingue-exolingue (Boyer, Plurilinguisme), dans laquelle les locuteurs mettent en œuvre diverses stratégies pour réduire les divergences codiques, sans recourir à une tierce langue. Du côté du partenaire fort, le procédé principal est bien entendu la simplification. C'est exactement ce à quoi nous avons assisté lors du cours de français dispensé aux légionnaires à deux semaines d'instruction. Consignes données par le lieutenant: «Question? Pas question ? », « Toi compris ? », « Lui fait correction. Si camarade erreur, lui corriger ». C'est donc cette langue simplifiée que les légionnaires apprennent. C'est en cela que la langue parlée à la Légion peut s'assimiler à un pidgin : absence de marqueurs de temps, adverbes utilisés comme marqueurs temporels, perte générale de la flexion, répétition du verbe pour indiquer une action répétée (Schumann), évitement de la subordination, paraphrase, morphologie verbale réduite à l'infinitif (Klein). Un lecte d'apprenant est défini comme le système d'expression d'un apprenant. Selon Wolfgang Klein, lectes d'apprenants et pidgins ont deux caractéristiques communes. En premier lieu, la distance entre les locuteurs : Klein entend bien sûr une distance sociale, puisque les pidgins apparaissent en premier lieu lors des échanges par exemple entre chefs coloniaux et indigènes. En second lieu, Klein remarque que ces deux systèmes linguistiques possèdent des traits des deux langues concernées, mais aussi des caractéristiques qui n'existent dans aucune des deux langues, ce qui se rapproche d'une interlangue. La situation linguistique à la Légion étrangère est celle où l'interlangue est continuellement renouvelée par de nouveaux engagés volontaires qui relancent le système de simplification là où un locuteur essayait de progresser, et devient alors une «variété de discours reconnaissable »(Whinnom). A., Russe, 15 ans de service :

«Mais nous, vu que on apprenne comme ça, donc on continue à parler comme ça. Vu que la légion c'est mouvement permanent, y en a toujours des gens qui arrivent, on essaie de parler à ce niveau des plus jeunes, que tout le monde qui comprenne.» 
Interlangue fossilisée (Selinker), ou interlangue historique (Boyer, Hybrides) : l'hybridation s'est fixée collectivement, donnant naissance au légiolecte. Pourtant, au milieu de ces tournures stéréotypées émerge l'influence de la première langue. C'est ce que nous allons aborder maintenant.

\section{L'hybridation linguistique dans l'interlangue des apprenants}

\subsection{Recherche et méthodologie}

Dans le passage d'une langue à l'autre, un locuteur est tiraillé entre deux langues en conflit et se trouve dans un fonctionnement diglossique. Ce dernier entraîne des marques transcodiques (Boyer, Plurilinguisme), c'est-à-dire des indicateurs du passage d'un code linguistique à un autre, ou de la présence de deux ou plusieurs langues dans le répertoire des locuteurs. Nous chercherons ici les marques transcodiques du passage du russe ou du polonais vers le français dans les interviews et les comptes rendus écrits de quelques légionnaires. Notre recueil de données s'articule autour de plusieurs corpus avec le protocole suivant :

- $\quad$ une interview dans la langue première de la recrue pour établir son parcours scolaire et universitaire, et connaître les autres langues éventuellement apprises. Cet entretien est destiné à établir le portrait d'apprenant du locuteur, en prenant en compte cependant la remise en question de la notion de «profil d'apprenant» dans les recherches sur l'acquisition de langue étrangère (Véronique);

- $\quad$ des tests institutionnels de FLE, de A1 à B1 (le niveau B1 ayant été retenu car il correspond au niveau exigé pour la naturalisation) ;

- un entretien en français sur la vie régimentaire et sur l'apprentissage du français par la recrue ;

- le récit à l'oral d'un film d'animation humoristique ;

- $\quad$ le récit écrit sous forme de compte rendu militaire de la vidéo racontée juste avant.

Ce sont les corpus décrivant la vidéo qui sont présentés ici. Le film présenté aux recrues dure $1 \mathrm{mn} 52$ et a pour titre Blow Me a Kiss. Il met en scène un jeune homme attendant le bus dans un lieu vide (un désert ?). Un chien arrive, ainsi qu'une vieille femme qui tente de se rapprocher du jeune homme afin de l'embrasser. Celui-ci lutte pour ne pas se laisser envahir et la vieille femme et lui finissent par se battre tant cette dernière est insistante. Finalement, le jeune homme est sauvé par l'arrivée du bus. Lorsque le bus démarre, il voit la 
vieille femme se rabattre sur le chien pour l'embrasser et se transformer alors en une créature sublime. Les légionnaires doivent raconter ce petit film à l'oral avec la consigne suivante : «Raconte ce film à quelqu'un qui ne l'aurait pas vu», puis par écrit, sous la forme d'un compte rendu qu'ils feraient à leurs supérieurs, avec la consigne : «Écris le compte-rendu à ton capitaine comme si c'était à toi que cette histoire était arrivée ». Bien que les corpus écrits constituent sans doute les données les plus intéressantes sur le plan de l'influence de la première langue dans le domaine de l'écrit, les corpus oraux sont également inclus, car ils présentent un intérêt certain pour attester de l'hybridation linguistique, dans la mesure où le discours est moins surveillé que dans les comptes rendus écrits.

Concernant la transcription des données provenant des corpus oraux, nous avons fait le choix de ne pas utiliser les conventions de transcription des interactions, celles-ci n'étant pas pertinentes dans l'étude de l'hybridation franco-slave. Concernant les récits, ne figureront ici que les tournures analysées, le format imposé de l'article ne permettant pas la reproduction intégrale des entretiens. Les exemples sont donc choisis pour leur adéquation aux axes de recherche que sont les marques transcodiques et l'hybridation linguistique. Au stade actuel de notre recherche, le corpus analysable à ces fins est le suivant : les comptes rendus écrits et oraux de deux légionnaires russes ayant respectivement quatre et quinze ans de service, le compte rendu écrit d'un légionnaire russe ayant six ans de service, les récits oraux de deux légionnaires polonais servant depuis trois ans et quinze ans de service, et le compte-rendu écrit d'un légionnaire russe de sept ans de service.

\subsection{Les énoncés des apprenants}

Nous commencerons ici par reproduire les énoncés des apprenants, dans lesquels les structures analysées figurent en gras. Nous procèderons ensuite à l'analyse des formes.

I., 30 ans, Russe, 6 ans de service, compte rendu écrit :

"J'ai honneur vous faire rendre compte, à 18 août 2013, j'était sur arrete de bus numero 23.

Je suis assi et pense de ma future. Et comme par assart est venu une femme l'agé. Elle voudra se presente, mais je ne pas envie de sa. Et, au bout moment, est arrivé le bus. J'ai pris le bus, j'ai fermé le port et laisse le femme avec ma chien sur place. Et dans quel que minut femme pris le chien sur le main et fais le bissous. Et j'ai vu avec mes seux propre, cette femme elle bien changé. Elle devenir une jeune fille. Mais, je ne regrette pas. Je suis parti dans le bus et je suis parti au quartier. En rentrant, je fais rendu compte a mon chef section. »

A., Russe, quatre ans de service, compte rendu écrit :

"J'ai d'honneur de vous rendre compte que j'ai rencontre une famme sur l'arrete bus, que voulu me bisou porscue elle a voulee change sa vie. En effet, je ne se vai pas que après le bisou elle devient une belle et je la refuse. Puis 
j'ai pris une bus et j'ai vui que elle a fait le bisou avec une chaine. La madam tout suit se change et devenue une belle.»

Le même légionnaire, récit à l'oral :

"C'est une histoire qui nous rencontre que c'est... pas, tout est pas bon qu'est ce qu'on voit. La femme elle voulait baiser un homme parce qu'alors ça se voit qu'elle a connu que dès qu'elle a fait le bisou elle va se changer. Donc elle va être une belle femme, ben voilà. L'homme bien sûr il a pas compris il a vu une vieille moche et donc il est parti. »

A., Russe, 42 ans, 15 ans de service, compte rendu écrit :

"J'ai l'honneur de vous rendre compte de faits suivants : 3 septembre 2013 à $12 \mathrm{~h} 30$ j'ai été à l'arret de bus " 19 » à côté de petit village. Un moment donné une veille dame qui se retrouver proche de moi à essayer de m'embrasser. Je resistais avec tout mes forces et j'ai reussi à monter dans le bus sans se faire toucher par cette veille. En revanche, ma chien à rester avec elle. En partant de cet endroit j'ai vu, que la veille femme à embrassé ma chien et elle se transformé à une très joli fille. J'ai essaye de sortir de bus mais en vain. »

Récit oral de R., 29 ans, Polonais, 3 ans de service.

"C'était histoire vraiment interessant. C'était pareil comme nous sommes maintenant. Malheureusement il y a pas de chien. Un mec qui attend pour bus. Il a tourné à gauche il a vu une femme qui était aussi tout seul, comme lui. Mais la femme qui voulait parler avec lui il voulait pas. En fait euh... A coté c'était un chien, après le bus qui s'arrive, il entré dans un bus, il parti, après .... Quand il rentre il a vu que elle a commencé à parler, parler, on peut dire avec le chien, après elle a se changer comme princesse. En fait il était regretter parce qu'il avait rien à foutre pour parler avec elle, pour rencontrer. Même elle voulait donner le bisou. C'était fini comme ça il est parti, elle est restée avec le chien. (...) Elle était nulle à chier. Elle avait pas un chose qui sont interesse pour lui.

Non, un chose, le cheveu, son cheveu qui était un peu tourné, comme toi. »

S, 36 ans, Polonais, 14 ans de service, récit oral :

«(...) La dame qui commence approcher trop vers lui sur arret de bus (...) et le chien qui boyait pendant tout ce moment là il reste avec cette dame là. Et après le garçon il regarde par la fenêtre comme la dame elle embrasse le chien. La dame elle se transforme contre une jeune fille. »

A, 30 ans, Polonais, 7 ans de service, compte rendu écrit :

«J'ai honneur de vous rendre compte que le jour 12 novembre 2013, je te sur une orette des bous. Entrant de dormir j'ai été reveille par un chaine et une vieille dame quel essei de me ambras. En effait comme le bus il arrive sur le zone. [...] Je remarque, après entre dans le bus que, le femme commanse ambrase le cheine ils se transforme en femme magnifique. » 


\subsection{Analyse des formes}

\subsubsection{Marques lexicales : stratégies de communication dues à un défaut de connaissance du lexique}

- fais le bissous / fait le bisou : visiblement, les recrues soit ne connaissent pas le verbe « embrasser», soit ne savent pas l'utiliser, et cherchent donc une autre façon de le dire. Parmi les interviewés, beaucoup utilisent régulièrement le verbe «faire » suivi d'un nom lorsqu'ils ne connaissent pas le verbe adéquat, ou ont tendance à le placer de façon presque systématique devant un autre verbe, par un processus de surgénéralisation (Selinker, Tarone), car ils ont remarqué les nombreuses occurrences des expressions françaises formées avec le verbe « faire ».

- elle voulait baiser: le verbe «embrasser», peu souvent utilisé dans la vie régimentaire, pose décidément problème. Ici, le légionnaire cherchait peut-être à produire une occurrence soignée de la langue cible, sachant qu'une tournure avec le mot «bisou » n'était pas satisfaisante, et produit cette tournure d'hypercorrection ou de surélaboration.

- donner le bisou : une variante de la forme «faire le bisou ». Les constructions avec le verbe «donner » n'étant pas aussi fréquentes que celles construites avec le verbe « faire», il convient mieux ici de désigner cette tournure comme périphrase que comme surgénéralisation.

- me ambras : ici la tournure attendue de l'infinitif « elle essaie de m'embrasser» n'est pas connue, ni l'élision devant l'initiale vocalique, mais le verbe «embrasser » semble familier du légionnaire.

- elle va se changer : au lieu de « elle va se transformer», le verbe «se transformer » n'étant visiblement pas connu de la recrue.

- le cheveu, son cheveu : une interrogation quant à l'utilisation du singulier pour « les cheveux »; l'équivalent polonais est également pluriel, comme en français : włosy. Si le locuteur voulait dire « la chevelure », alors l'utilisation du singulier à deux reprises est une marque transcodique lexicale.

L'analyse des formes lexicales met en évidence un point commun chez les recrues : l'ignorance du verbe «embrasser », dont on trouve la première occurrence chez un locuteur de sept ans de service, la plupart utilisant des constructions avec le verbe «faire» ou « donner ».

\subsubsection{Calques venant de la structure syntaxique de la langue première}

- sur arrete de bus/ sur l'arrete de bus/ sur une arrete de bus/ sur arrêt de bus : seul le locuteur russe de quinze ans de service utilise la préposition « à » pour parler de l'arrêt 
de bus. Tous les autres utilisent «sur », preuve d'un calque syntaxique de la langue première. En russe, être à l'arrêt de bus (быть на остановке) utilise la préposition « на », traduite presque toujours en français par «sur». Même phénomène en polonais : na przystanku. Ce calque dans l'interlangue des recrues semble tenace : seule la recrue russe de quinze ans de service écrit «à ». Les données actuelles ne sont pas assez quantitatives pour tirer des conclusions. Une précision s'impose toutefois : le locuteur russe qui utilise la préposition « à », a précisé lors de l'interview qu'il avait beaucoup travaillé seul en langue française. Il faudrait interroger d'autres locuteurs moins avancés que lui, mais avec autant d'années de service, pour observer les occurrences de cette préposition et déterminer si cette structure est fossilisée dans l'interlangue des locuteurs slaves ou non.

- ça se voit qu'elle a connu : la recrue voulait dire « apparemment, elle savait » pour signifier que la vieille femme savait qu'elle se transformerait si elle embrassait quelqu'un. Il s'agit ici d'une traduction littérale de l'équivalent russe видимо она знала composé de la tournure impersonnelle видимо construite sur le verbe видеть : voir.

- elle va se changer : nous avons déjà mentionné cette forme dans la partie précédente consacrée aux marques lexicales, mais cette tournure doit figurer ici également. En effet, en russe, le verbe «changer» est pronominal : измениться. Le locuteur reproduit donc la pronominalisation du verbe en langue cible.

- j'ai vu, que la vieille femme : en russe, la virgule est obligatoire entre la proposition principale et toute proposition subordonnée relative, complétive ou circonstancielle. Le locuteur reproduit ici cette obligation comme la phrase russe l'exigerait: Я видел, как старая женщина. Nous considérons donc ici que la ponctuation fait partie de la syntaxe, dans la mesure où cette obligation touche à l'organisation de la phrase complexe, c'est-à-dire à la syntaxe de la phrase.

- qui attend pour bus : il s'agit d'un calque syntaxique du polonais czekać na autobus, langue dans laquelle le verbe «attendre » est suivi de la préposition «na »dont l'une des nombreuses acceptions est « pour». Ce verbe induit une construction indirecte et le locuteur met ici un complément d'objet indirect en lieu et place du complément d'objet direct attendu.

- il regarde par la fenêtre comme : Ici, le légionnaire veut dire « il voit par la fenêtre que la dame embrasse le chien » et traduit littéralement du polonais : patrzy przez okno, jak pani caluje psa. En polonais, jak introduit une proposition que le français introduirait par «que », mais en dehors de cette utilisation, il est le plus souvent traduit par « comme» ou 
«comment » en français. Il s'agit donc ici selon nous d'un calque plutôt syntaxique et non lexical.

- une femme l'âgé : ici le l'adjectif est traité comme un substantif et le locuteur place un déterminant devant, faisant preuve d'une surgénéralisation, où une règle de la langue cible connue est généralisée (Faerch et Kasper).

- prendre sur la main : traduction littérale par transfert de l'expression russe «prendre dans ses bras », взять на руку, qui se traduit littéralement en français par «prendre sur la main », avec à nouveau la préposition $\mu a$, « sur ».

\subsubsection{Marques morphologiques touchant au genre}

- ma chien (x3) / une chaine : l'intégralité des locuteurs russes utilise ici le féminin pour désigner le chien. En russe, les termes génériques pour désigner les animaux sont, à quelques exceptions près, tous féminins, contrairement au français. On retrouve ce calque de quatre ans à quinze ans de service ; il semble donc fossilisé dans l'interlangue des locuteurs russes. Les Polonais interrogés, quant à eux, utilisent bel et bien le masculin, le mot « chien » étant dans leur langue masculin également.

- un chose : « un » au lieu de « une » devant le nom « chose» : cette erreur de genre s'explique ici car l'équivalent polonais est lui aussi masculin : rzecz.

\subsubsection{L'hybridation « Légion »}

Nous avons regroupé ici les expressions relevant de la langue parlée à la Légion.

- vous faire rendu compte / fais rendu compte / j'ai d'honneur de vous rendre compte / j'ai honneur de vous rendre compte : il n'y a ici aucun calque notoire de la langue première en français, mais une utilisation erronée de la structure attendue par le commandement pour les comptes rendus militaires devant commencer par « J'ai l'honneur de vous rendre compte que....». La seule recrue écrivant correctement cette tournure est le légionnaire russe de quinze ans de service.

- il avait rien à foutre pour parler avec elle / elle était nulle à chier : Ces expressions fleuries sont des modèles préfabriqués (Tarone) utilisés par tous les légionnaires jusqu'à ce qu'ils en comprennent le degré de vulgarité, ce qui peut prendre un certain temps s'ils ne communiquent que rarement avec des civils. Dans l'interlangue de la jeune recrue, ce qui est bien est désigné par les expressions « nickel » ou «boule de feu » et tout ce qui n'est pas bien est «nul à chier ». Il n’y a pas de segmentation en mots dans ces expressions et les légionnaires sont souvent mortifiés d'en apprendre la traduction littérale et de se rappeler des occasions où ils l'ont utilisée en ignorant le registre de langue. Reprenant la définition donnée par Hakuta en 1976, Elaine Tarone décrit le modèle préfabriqué comme un segment du 
discours régulièrement modélisé, précisant qu'il est utilisé sans connaissance de sa structure sous-jacente. Nous sommes bel et bien dans ce cas de figure ici.

- magnifique : cet adjectif fait partie du premier bagage linguistique du légionnaire, avec «boule de feu» et «nul à chier». Est «boule de feu» tout ce qui se rapporte positivement au domaine guerrier, aux relations entre camarades, aux performances sportives. «Magnifique » est employé pour parler des filles, de la cuisine, du temps qu'il fait, et apparaît très tôt dans les interviews des légionnaires (premières occurrences dès huit mois de service).

- il arrive sur le zone : ici, ce n'est pas l'erreur de genre sur le substantif «zone » qui nous parait le plus intéressant, mais l'utilisation du terme «zone» dans ce contexte. Les récits des légionnaires font souvent état de situations dans le civil où ils emploient un terme inapproprié par ignorance des différents registres. Une de ces situations racontée par le légionnaire polonais de sept ans de service dans son interview est celle d'une recrue accostant une femme dans un bar. Voulant lui demander où elle habite, il lui aurait dit : «C'est où ton bivouac?» Lors du cours de tactique que nous avons eu l'occasion d'observer à Castelnaudary, le lieutenant francophone expliquait le vocabulaire nécessaire à la compréhension de l'exercice qui allait suivre à des recrues de six semaines de service avec le jargon militaire/légionnaire : il y était question de bivouac, de guitounes (les tentes), de kékés (les buissons), de piste (la route). Les légionnaires connaissaient ces mots. Lorsque le lieutenant leur a demandé les équivalents civils de ces mots, ce fut un silence opaque. Seuls les francophones connaissaient les correspondances civiles des mots. En revanche, lorsque le sergent originaire d'Amérique du sud prend la relève pour expliquer l'exercice, on retrouve la langue «naturelle » des légionnaires avec son jargon et sa syntaxe simplifiée :

"Lieutenant il a expliqué, il a facilité pour que vous rendre compte. C'est bon? C'est pour vous faciliter pour caporal pour expliquer pour sergent qu'est-ce qui se passe. Visuel avec découvert, visuel avec piste, visuel avec guitoune, kékés. C'est bon lieutenant il a facilité. Bon, sergent il va expliquer mission. Deux types de mission aujourd'hui on va voir. Mission avec ennemi, mission sans ennemi. Mission pas ennemi, no ennemy. »

Les interviews des légionnaires sur leur parcours à la Légion ou sur leur vie régimentaire sont plus riches en expressions stéréotypées que les récits oraux ou écrits du petit film, qui induisent un autre vocabulaire que celui strictement militaire. Nous voyons ici que certains automatismes ou modèles préfabriqués y apparaissent malgré tout. 


\section{Conclusion}

Le français appris à la Légion étrangère est une langue à part. Par le contexte d'acquisition plurilingue et exolingue avec interdiction d'utiliser sa première langue, elle transmet un input tout à fait particulier aux recrues: langue simplifiée, expressions stéréotypées, structures erronées rendues pérennes par le renouvellement permanent des recrues. L'accès difficile à la vie civile dans les premières années vient renforcer l'ancrage de cette langue. Nous avons tenté de montrer ici un aperçu de l'influence de la langue première et du contexte d'acquisition dans la langue cible. Certaines interviews montrent en outre une interlangue dans laquelle l'influence d'autres langues apprises avant le français se fait sentir. En effet, peu de locuteurs ont un répertoire linguistique ne comportant qu'une seule langue étrangère. Concernant les dimensions psychologiques de l'apprentissage de la langue, les témoignages tendent à montrer une certaine résistance. Nous l'avons vu dans les interviews de deux soldats, les motivations à apprendre la langue peuvent être sérieusement émoussées par les punitions physiques et l'interdiction d'utiliser sa langue. Il faut apprendre vite, pour comprendre les ordres donnés et ne pas «ramasser». Refuser d'apprendre le français ou déroger systématiquement à l'interdiction de la première langue constitue alors un acte de rébellion, une tentative de conserver son identité et de ne pas marcher au pas Légion.

\section{Bibliographie}

Boyer, Henri. Plurilinguisme : «contact»ou «conflit» de langues ? Paris : Harmattan, 1997.

---. Hybrides linguistiques : genèses, statuts, fonctionnements. Paris : Harmattan, 2010.

Faerch, Claus et Gabriele Kasper. «Plans and Strategies in Foreign Language Communication ». Strategies in Interlanguage Communication. Londre/New York: Longman, 1980. 20-60.

Klein, Wolfgang. L'acquisition de langue étrangère. Paris : Armand-Colin, 1989.

Porquier, Rémy. «Deux repères de recherche sur l'interaction exolingue ». Linx 49 (2003). http://linx.revues.org/538. En ligne, consulté le 10 octobre 2012

Schumann, John H. «Implications of Pidginization and Creolization for the Study of Adult Second Language Learning ». New Frontiers in Second Language Learning. N.p. : Newbury, 1972. 137-151.

Selinker, Larry. «Interlanguage ». New Frontiers in Second Language Learning N.p. : Newbury, 1972. 114-136. 
Tarone, Elaine, A. D. Cohen et G. Dumas. «A Closer Look at some Interlanguage Terminology : A Framework for Communication Strategies ». Applied Linguistics and Language Studies. N.p. : Somerset, 1983.

Véronique, Daniel. «Quel profil d'apprenant? Réflexions méthodologiques ». Acquisition et interaction en langue étrangère 4 (1994). http://aile.revues.org/1256._En ligne, consulté le 15 septembre 2011.

Whinnom, Keith. «Linguistic Hybridation and the 'Special Case' of Pidgins and Creoles ». Pidginization and Creolization of Languages : Proceedings. Dir. Dell H. Hymes. Cambridge : Cambridge UP, 1971 : 91-116. 